\title{
Les usages des expertises psy au procès d'assises et les définitions pratiques de la responsabilité*
}

\author{
Sébastien Saetta \\ François Sicot \\ Tristan Renard \\ LISST - Université de Toulouse 2 \\ INSIDE - Université \\ du Luxembourg
}

La question de l'évaluation de la personnalité des accusés, qui accompagne celle de l'individualisation des peines, est au centre des préoccupations du courant désigné sous le terme de "défense sociale nouvelle». Cet article s'intéresse à la performance de l'expert dans un contexte particulier, celui des audiences de cours d'assises, où la parole de l'expert est encastrée dans un enchaînement de séquences et où la personnalité de l'accusé est abordée par une pluralité d'acteurs. Dès lors, les échanges autour de cette question ne visent pas tant à définir une dangerosité ou une curabilité que l'élément moral du crime. Ce regard sur l'expertise en acte montre que, dans ces procès, les acteurs construisent des définitions pratiques de la responsabilité.

Depuis 1998 («Loi 98468 du 17 juin 1998 relative à la prévention et à la répression des infractions sexuelles ainsi qu'à la protection des mineurs»), en France, de multiples lois et rapports sont rédigés et publicisés qui modifient les mesures pénales et les dispositifs relatifs aux criminels dangereux et/ou malades mentaux. Dans le cadre d'un numéro de revue consacré à la défense sociale, il n'est pas inutile de commencer par relever que la création des USHA (Unités Hospitalières Spécialement Aménagées), les modifications dans la mise en œuvre de l'injonction de soins, les nouvelles fonctions prêtées aux expertises psychiatriques, la sécurisation des hôpitaux psychiatriques, les différents projets de structures fermées sont considérés comme des mesures de sûreté et, «partant», de défense sociale. Dans le même mouvement, ce qui est critiqué dans ces lectures des réformes, c'est leur antihumanisme qui rabat certains hommes au rang de « prédateurs ${ }^{1}$, les considère comme déterminés et définitivement dangereux.

* Ce travail rentre dans le cadre d'une recherche financée par le Fonds National de la Recherche (FNR) du Luxembourg, par le biais d'une Allocation Formation Recherche (AFR).

1 En octobre 2009, un individu accusé de l'enlèvement et du viol d'un enfant est qualifié de prédateur froid, sadique et incurable par un expert psychiatre. En 2007, N. Sarkozy tenait les pédophiles pour des prédateurs d'enfants et se déclarait incliné à penser qu'on naît pédophile. 
Cette assimilation des réformes en cours à des mesures de défense sociale amalgame différentes périodes du mouvement de défense sociale relativement hétérogènes. La défense sociale nouvelle refuse le déterminisme ${ }^{2}$ qui, de fait, inspire ces récentes réformes, et insiste au contraire sur la nécessaire prise en compte à la fois de la personnalité du criminel et du milieu social pour déterminer, individualiser la peine. L'évaluation de la personnalité des accusés et des condamnés joue un rôle essentiel dans ce mouvement de politique criminelle humaniste. En 1952, lorsqu'il édite sa communication sur l'observation médicopsychologique et sociale des délinquants, $M$. Ancel discute des modalités de l'introduction, selon lui indispensable, de cette observation dans le procès pénal.

Dans cet article, nous examinerons ce qu'il en est, aujourd'hui, de cet examen de la personnalité au procès d'assises. Qu'est-ce que l'expertise psychiatrique judiciaire? Et particulièrement, quelle place prennent les expertises «psy» ${ }^{3}$ dans le jugement? Comment les acteurs du procès procèdent-ils, avec les «psy», pour évaluer la personnalité des accusés? Plus encore, pour quoi les «psy» sont-ils requis ? On pourra lire également ces analyses comme une contribution à la description d'une pratique psychiatrique et de ses usages sociaux.

Afin d'avancer sur ces différentes questions, nous verrons d'abord en quoi consiste l'étude de la personnalité lors du procès d'assises et quel rôle y joue l'expert psychiatre aux côtés des autres acteurs. Nous montrerons que l'analyse des échanges langagiers est indispensable pour comprendre ce qui se dit sur la psychologie et la personnalité de l'accusé, et que les rapports écrits des experts ne prennent qu'une place limitée dans la dynamique du jugement en train de se faire. Dans un second temps, nous dégagerons de l'analyse de ces échanges les différents aspects de ce qui constitue le cœur du travail mené avec les experts : la recherche de la responsabilité morale de l'accusé. En effet, loin qu'ils ne soient requis que pour évoquer la responsabilité pénale, la dangerosité ou la curabilité de l'accusé, les experts sont conviés à éclairer le jury sur les questions de savoir si l'élément moral du crime est constitué, si la personnalité rend l'accusation plausible et si l'accusé est capable de travailler sur lui-même.

La démonstration repose sur l'observation de 16 procès d'assises ${ }^{4}$ qui se sont tenus dans cinq tribunaux relevant de trois cours d'assises de la France, sous cinq présidences différentes. Certains de ces procès se tenaient en première instance, d'autres en appel. Ils ont concerné des affaires de vols, viols, dégradations, homicides, coups et blessures et leurs différentes déclinaisons juridiques. Après avoir observé les premiers procès dans leur totalité, nous avons repéré les séquences principales au cours desquelles étaient évoquées et discutées les expertises «psy». Dans l'impossibilité légale d'enregistrer les débats, nous avons noté le plus exhaustivement possible les verbatim des échanges impliquant les expertises «psy».

2 Le positivisme est par essence déterministe. Or, le mouvement de défense sociale rejette aussi bien le fatalisme biologique dérivé de Lombroso que le causalisme social inspiré de Ferri et se montre très réservé sur les «prédispositions congénitales» ou la catégorie des «pervers constitutionnels (Ancel, 1983, 50).

3 On verra infra la justification de cette appellation qui renvoie à un ensemble de considérations sur la psychologie des accusés. Ce regroupement est justifié par l'observation et l'analyse des procès.

4 Une part de ces procès se déroulant à huis clos, nous avons demandé au président d'assises l'autorisation d'être présent. 


\section{Le travail des experts en acte et le langage dans l'action}

Dans la première page de l'ouvrage qu'il a dirigé en 2003 sur l'expertise, F. Chauvaud rapporte une scène citée par un chroniqueur judicaire et qui concerne un expert officiant dans la première moitié du XX $\mathrm{X}^{\mathrm{e}}$ siècle: Cétait un cas de strangulation. En entrant dans le prétoire, il avait aperçu, au banc de la presse, un journaliste ami qui, ostensiblement, portait la main à son cou. Sans doute pensa-t-il, le légiste local qui m’a précédé n'a-t-il pas été suffisamment précis. Et on veut m'en avertir. Le voici qui décrit la position des doigts du meurtrier autour du larynx de la victime, indique le temps de serrage... l'accusé était blême; le défenseur aussi de colère. Mais docteur, expliqua le journaliste après l'audience, je vous faisais signe, simplement, que votre épingle de cravate était mal ajustée. Et F. Chauvaud de relever que l'anecdote illustre l'importance de l'expertise. À nos yeux, elle illustre bien autre chose: l'importance du contexte dans lequel officie l'expert c'est-àdire non seulement le prétoire mais cette cour d'assises, avec ce président, ce jury, ce public ou non s'il y a huis clos, cette organisation des échanges entre professionnels, experts, témoins. La performance de l'expert se déroule dans des contextes bien précis et c'est là qu'elle prend du sens, se développe plutôt d'une manière ou d'une autre et, finalement, en vient à participer au jugement.

Deux autres données incitent le chercheur à aller observer le travail des experts en acte, lors du procès. On sait l'importance que revêt, en France, l'oralité des débats et des dépositions dans le fait social de rendre justice et particulièrement dans le procès pénal. Des observations classiques, qui ne retiendraient pas la dynamique des échanges discursifs manqueraient l'essentiel. D'autre part, les membres du jury d'assises n'ont pas accès aux pièces écrites du dossier et ils n'auront à connaître des expertises que ce qui sera lu des rapports, ce qui sera présenté et discuté lors du procès. C'est sur cette base qu'ils formeront leur jugement, décideront de la culpabilité et de la peine.

Lors des procès d'assises, les psychiatres et les psychologues ne lisent pas leurs rapports dans leur intégralité ${ }^{5}$. Ils n'en lisent que certains passages, en proposent un résumé, une présentation vulgarisée et/ou s'appuient sur des notes élaborées à partir de rapports rédigés plusieurs mois, voire plusieurs années auparavant. Ce que disent les experts de la personnalité n'est que pour partie contenu dans le rapport qu'ils ont rédigé pour l'instruction. Tandis que les écrits sont organisés selon un plan standard, chaque expert suit, lors de sa prestation, un plan qui lui est propre et qui laisse plus ou moins de marges à des développements absents du rapport ou à des improvisations conjoncturelles, qu'elles soient liées aux débats en cours lors du procès, à des événements qui se sont produits dans le pays ou à des préoccupations présentes de l'expert. Suite à cette présentation, les experts répondent à des questions dont les réponses ne sont pas nécessairement dans les écrits parce qu'elles concernent l'apport de tel test, ou ce que l'on sait en général de tel crime ou de telles personnalités ou ce qui vient d'être discuté lors de l'audience.

Pour comprendre la place des expertises psychologiques et psychiatriques dans le jugement pénal, il est donc nécessaire d'observer les échanges autour des expertises lors des audiences. Partant de ce constat du rôle crucial de l'énonciation, on emprunte ici à des

5 La performance orale lors du procès est d'ailleurs pour les magistrats un critère majeur de la définition du bon expert. 
perspectives sociologiques qui étudient ce que les acteurs font vraiment, par exemple dans un environnement judiciaire, à l'occasion de leurs face à face interactionnels et particulièrement leurs actes de langage $e^{6}$. Ce qui justifie la méthodologie et la perspective théorique adoptée est donc une réalité sociale: le fait que les jurés ne connaissent du dossier, des expertises que ce qui en est évoqué au procès, et que les discours des experts constituent une performance au sens de la linguistique (ensemble des énoncés produits). On ne peut étudier les expertises «psy» dans la production du jugement qu'en situant la performance des experts autour de leurs écrits dans le contexte de leur énonciation.

Que font les acteurs ensemble lorsqu'ils échangent avec les experts autour de la personnalité des accusés? Dans quel travail les participants sont-ils engagés et quels sont les mécanismes conversationnels qui leur permettent de le réaliser? En quoi ces échanges participent-ils à la formation d'un jugement? On s'attache dans cet article au droit et à la psychiatrie en action et l'on cherche à décrire ce qui est fait par ce qui est dit: comment les membres mobilisent le langage pour réaliser le travail interactionnel en cause, ici le jugement pénal.

Pour comprendre comment les acteurs progressent collectivement sur un certain nombre de questions - Était-ce un viol? La consommation d'alcool est-elle une circonstance aggravante du crime? L'accusé était-il parfaitement conscient des conséquences de ses actes? - et donc quelles définitions pratiques ils produisent du viol, du crime, de la conscience, il y a lieu de décrire comment les énoncés s'agencent dans des tours de parole, quelles sont les procédures rhétoriques utilisées, ce qu'il en est de l'énonciation et non seulement des énoncés.

Il est ainsi notable que les énoncés des experts sont pris dans un ensemble d'énoncés qui les précèdent ou les suivent et forment ensemble une grille de lecture des faits et des personnalités. Les questions posées par le président ou les parties dépendent en partie de ce qui a été échangé précédemment et l'expert lui-même peut construire sa performance en fonction de ce qui a été dit avant lui (je ne vais pas répéter ce qu'a dit mon collègue/ ${ }^{7}$ Comme il a été dit tout à l'heure/Comme je passe en dernier, je vais développer des éléments jusqu'ici peu abordés). À l'oral, pressé de répondre aux questions de la cour, l'expert improvise à partir de son expérience ou de ce que l'on sait pour ce genre de personnalité ${ }^{8}$.

6 Nous ne cherchons pas à faire une ethnométhodologie ou une sociologie pragmatique de l'expertise psychiatrique. Ce sont un certain nombre de ces postulats de départ qui sont partagés et des outils qui sont mobilisés. En particulier l'idée que le sens des phénomènes doit être considéré comme un phénomène public, qui ne requiert ni posture mentaliste, ni supputations sur ce que les acteurs cherchent à faire - leurs intentions - ni théories pour expliquer ce qui est dit. En outre, le droit et la psychiatrie en acte dans les procès ne sont pas considérés comme des versions défectueuses du droit ou de la connaissance scientifique. Pour une présentation des applications de l'ethnométhodologie et de l'analyse de conversation au champ juridique, cf. le numéro spécial de Droit et société, 2001, ainsi que Travers, Manzo, 1997. Pour une application de la sociologie pragmatique à l'analyse de procès judiciaires: Ughetto, Weller, 2008. Pour une approche pragmatiste de l'expertise médicale, Dodier 1993. Cet auteur critique les approches qui, sur ce sujet, présentent l'inconvénient de porter un verdict épistémologique. Il s'agit selon lui d'approches dualistes, préoccupées de faire le partage entre ce qui est proprement interne au jugement médical et ce qui lui est externe», et qui présentent la particularité de partir "d'un modèle de jugement autonome, un schéma épuré de raisonnement médical, juridique ou scientifique, et de confronter les activités empiriques avec ce modèle (Dodier, 1993, 21). Par ailleurs, sur les actes de langage comme objet sociologique, Borzeix, Fraenkel, 2001.

7 Dans la suite de l'article, les ponctuations suivantes accompagnent les énoncés. Le / sépare des énoncés décontextualisés, qui peuvent être extraits de différents procès. Les [] apportent une précision contextuelle des sociologues sur l'action. Les (...) signalent une coupe dans les propos et les ... une phrase laissée en suspens.

8 Des experts parlent à ce propos d'exercice de haute voltige. Le flux des échanges n'exclut donc pas ce qui constitue a minima des pertes de contrôle des propos. Nous n'excluons évidemment pas l'existence de jugements de valeurs, 
L'ensemble des énoncés prononcés lors du procès constitue le contexte indispensable à la compréhension de chaque énoncé particulier. S'il est indispensable de ne pas isoler un énoncé de ce qui le suit ou le précède immédiatement, des tours de parole proprement dit, l'analyse d'une séquence isolée n'est pas a priori pertinente. En effet, les acteurs ne soulignent pas nécessairement ce contexte par des formules du type: comme vous avez pu le voir la veille ou, comme on le verra par la suite, néanmoins, ceux qui parlent peuvent se référer à ce qui a été dit deux jours auparavant. Quant aux jurés, par définition, ils ont à l'esprit, lorsqu'ils entendent un énoncé, la totalité des échanges. Cette totalité réalise une structuration globale de la conversation. C'est pourquoi, dans nos analyses, nous cherchons à déterminer au cas par cas le contexte pertinent du sens d'un énoncé qui peut être aussi bien l'énoncé immédiatement précédent qu'un énoncé du jour précédent.

Un procès pénal est constitué d'un ensemble complexe d'encastrements d'énoncés d'origines diverses, oraux ou écrits, présents et passés. De manière exemplaire, l'acte d'accusation, qui peut comporter une dizaine de pages et dont la lecture par le greffier peut durer une demi-heure, propose une première mise en récit, un scénario du crime, à partir de pièces du dossier. Des éléments y sont agencés, des extraits cités de manière à donner à entendre une cohérence. La forme autant que le fond de ce texte participe au processus de construction de la vérité juridique: Ils ont nié être les auteurs de ce braquage. Néanmoins un témoin entendu... L'utilisation de l'imparfait de l'indicatif - qui situe l'événement dans le passé - comme la multiplication des citations - citations de l'inculpé lui-même au juge ou citations pour ainsi dire au troisième degré par l'acte d'accusation qui cite l'expert citant l'accusé - produisent un effet rhétorique de vérité ${ }^{9}$. Le véhicule de dotation Renault Scenic était également en flammes. Les policiers subissaient d'importantes séquelles psychologiques. La forme du discours participe à la production de la factualité juridique (Ce 25 juin, je me transporte au tribunal). Enfin, on relèvera la fréquence importante de procédures de mise à distance du discours de l'autre: Son histoire telle qu'il nous la raconte... / Il dit que.../Il considère que...

Dans ces actes d'accusation, la totalité des expertises «psy» est ramassée en deux ou trois phrases tirées des écrits des experts sans qu'il ne soit possible de repérer une logique de la sélection par le magistrat. La construction d'un acte d'accusation implique inévitablement sélection, suppression et transformation.

Les acteurs du procès ne cessent d'opérer des emboîtements d'énoncés d'origines très diverses, de reprendre en reformulant ce qui a été dit ou écrit auparavant, d'alterner citations et re-présentations d'énoncés, opérant par là une mise en sens à plusieurs niveaux: ce qui a été dit, ce qu'il faut comprendre de ce qui a été dit, et donc ce qui est.

Par exemple un président d'assises déclare, lors d'une audience: L'une des sours dit, je lis [se penche vers le procès-verbal] : «mon frère est irrécupérable». Alors c'est avec beaucoup

de propos sexistes, racistes ou autres lors des procès. Simplement, dans la logique de notre analyse, ces jugements ne peuvent être isolés du contexte de leur énonciation et des effets qu'ils produisent.

9 Les citations directes sont un type de paroles rapportées minimisant le fossé séparant la décontextualisation et la recontextualisation de paroles précédentes. Elles font en sorte que les mots qui sont dits ici et maintenant apparaissent comme la réplique exacte des mots prononcés dans un contexte historique... Les citations directes, qui prétendent représenter les paroles dans leur formulation exacte, fonctionnent particulièrement bien comme moyen de construire dramatiquement le discours... et comme un outil permettant de fournir les preuves nécessaires à l'évaluation ou, mieux encore, à la construction de l'authenticité de l'affirmation (Matoesian, Coldren, 2001, 404-405). 
de prudence que je livre cette phrase aux jurés. Mais aujourd'hui ça entre en résonance avec votre expertise [cite un extrait de l'expertise] : le délit s'inscrit dans la poursuite des besoins immédiats. S'il est coupable vous dites que c'est un choix de vie. Donc le pronostic de réadaptation reste incertain. Ce faisant, il a cité, mis en contexte et donc en rapport deux extraits d'écrits différents. Il en propose une lecture à plusieurs niveaux: 1. Ça entre en résonance; 2 . On peut en conclure; mais 3 . Cette conclusion ne peut être définitive car elle repose sur une déclaration qu'il faut prendre avec prudence. Par contamination pour ainsi dire, c'est toute la conclusion qui doit être entendue avec prudence, ne doit pas être entendue comme autre chose qu'une simple hypothèse, le président ne pouvant, en droit juger.

Lors des audiences, les participants proposent fréquemment des relations de causalité. Mais ils peuvent le faire plus ou moins explicitement en évoquant une relation de causalité de type psychologique par exemple: Il va dériver vers des groupes ou des groupuscules type «skin», où il va pouvoir exprimer un certain nombre de choses. D'abord il va trouver une certaine place, ça lui permet d'exister un peu; il n'existe pas trop dans le regard de son père donc il va essayer d'exister dans ce groupe-là (expert) - soit « incidemment », par la simple opération de juxtapositions de propositions - Héroïnomane, Monsieur Ega [le père de l'accusé] met fin à ses jours en 1993 (enquêteur de personnalité).

Les acteurs les mieux pourvus en capital culturel manipulent les formules de rhétorique classiques (prolepses, litotes, périssologies, métonymies, hyperboles...) afin de produire des effets sur les auditeurs, le jury, au delà du sens premier des mots, afin de faire passer une idée sans en prendre l'entière responsabilité: effets de persuasion, de mise en valeur, d'insistance, de déplacement du sens, de connivence, de suggestion...

Sur cette scène publique que constitue le procès pénal, nombre d'interventions ont une dimension rhétorique en un autre sens. Les parties qui interrogent les témoins ou les experts s'adressent à travers eux prioritairement aux jurés. Leurs questions n'ont pas de sens dialogique, elles ont un sens collectif, d'exposition. En interrogeant, il s'agit de manifester le raisonnement adéquat pour juger: les questions que le jury doit se poser, les éléments dont il doit normalement disposer pour ensuite délibérer en toute connaissance de cause ${ }^{10}$.

Tant en ce qui concerne l'articulation des différents discours sur la personnalité que pour la fonction prêtée à l'expertise psychiatrique, nous pouvons distinguer trois types de procès. Dans le premier, l'expertise psychiatrique est tenue pour négligeable, aussi bien par le président que par les parties et les experts. Un consensus s'est implicitement forgé a priori: l'affaire ne relève pas de la psychiatrie en quelque manière. On parle bien de la personnalité mais avec ceux qui sont présents: témoins, enquêteurs de personnalité. La lecture rapide des expertises par les présidents étant souvent peu audible, c'est la parole de ceux qui sont présents qui retient l'attention de la salle. Dans le deuxième type, le moins fréquent, on se trouve en présence d'une abolition du discernement controversée ou d'une altération, les débats se focalisant dans le dernier cas sur le degré d'altération. Dans ces

Il n'est d'ailleurs pas rare que l'un des acteurs professionnels de la scène explicite ce qui se joue dans les échanges (par exemple, un président et un accusé: - Racontez moi votre travail: pas de chômage? - Alors quand j'ai commencé... - [Le président, coupant l'accusé]: Ne vous perdez pas dans les détails (l'important c'est de faire apparaître que vous travailliez). 
procès, on entre dans les débats d'experts et l'on cherche à savoir si l'accusé relève de la sanction ou s'il est pénalement irresponsable.

Dans le troisième type de procès, ni l'altération, ni l'abolition du discernement n'ont été retenues par les experts. On discute alors, avec les expertises psychologiques, psychiatriques, l'enquête de personnalité, l'interrogatoire du président, de l'adéquation de la personnalité et des faits incriminés d'une part, de la responsabilité subjective de l'accusé d'autre part. En substance, un président a pu exprimer, en se tournant vers le psychiatre, ce qui était en question dans ces échanges: Hier j'ai compris l'importance de son enfance, ses relations avec ses parents dans ses actes. Est-ce que ça a pu être déterminant? Quelle est la part de responsabilité de chaque individu dans sa destinée? Les analyses présentées dans cet article relèvent de ce troisième type de procès, de loin les plus nombreux.

\section{Le contexte et le président}

\section{Qui parle de psychologie et à quels moments du procès?}

Lors des audiences, les expertises psychiatriques ne prennent qu'une part relative et contingente dans l'examen de la personnalité des accusés. A priori, les expertises psychologiques et psychiatriques sont essentiellement évoquées en trois séquences: lors de la lecture de l'arrêt de renvoi au tout début du procès, lors de la présentation de leurs analyses et conclusions par les experts et, enfin, au moment du réquisitoire de l'avocat général et des plaidoiries des avocats de la partie civile et de la défense. Mais trois séries d'éléments viennent perturber ce schéma.

Premièrement, les experts ne sont pas toujours présents à l'audience. Il n'est pas rare que les experts se soient déclarés indisponibles. Dans ce cas, c'est le président qui lit l'expertise, en partie ou en totalité, assortissant parfois sa lecture de commentaires ${ }^{11}$. Ce qui exclut de facto les débats autour de ces expertises.

Deuxièmement, la personnalité est évoquée par d'autres que les experts. Le président tout d'abord lors de son interrogatoire de curriculum qu'il peut plus ou moins développer, assortir de considérations "psy» (Le décès de ce père vous a durablement marqué (...). Vous supportiez très mal votre beau-père (...). Ce comportement démontre un malêtre certain). L'interrogatoire de curriculum est la première occasion pour les jurés, d'entendre parler de la personnalité de l'accusé. Là encore, la liberté du président est grande qui peut par exemple choisir de donner plus ou moins de place au casier judiciaire, au comportement en détention (lorsque les prévenus étaient incarcérés ou jugés en appel), à l'enfance...

Elle est évoquée également par l'enquête de personnalité. En l'absence des experts, les propos des enquêteurs de personnalité prennent tout particulièrement du poids. Or, ceux-ci suivent un fil qui n’est pas si éloigné des expertises: ils commencent par résumer

11 Propos de présidents: Vous avez fait l'objet d'une expertise psychologique et d'une expertise psychiatrique, on va les lire [fait une lecture intégrale, rapide, peu audible, assortie de commentaires] Oui bon la justice appréciera/ Ça c'est du vocabulaire de psy ou je m'y connais pas/Attendez c'est pas fini, vous avez un dictionnaire?/C'est pas le premier qui nous parle de chauve-souris sur cette planche!/Sur cette expertise elliptique - on va essayer de ne pas être trop... véhément - est-ce qu'il y a des questions? 
une biographie de l'accusé puis apprécient sa personnalité, évoquent sa "psychologie»: Livré très jeune à lui-même, il n'a pas réussi à se construire d'interdits/Je n'ai pas décelé de troubles psychiques/Le milieu familial dans lequel il a grandi ne lui a certainement pas permis de s'épanouir.

Les différents avocats peuvent également intervenir dans ces échanges autour de la personnalité, soit pour demander des précisions sur tel terme technique (Vous avez parlé d'absence de modèle identificatoire, vous pouvez nous expliquer?) soit tenter eux-mêmes des «lectures psy» (Dans ses actes, il répète ce qu'a fait son père, ce père qui a lui a manqué. Il est accusé d'avoir commis ce braquage alors que son fils, qu'il a appelé comme son père, vient d'avoir 12 ans, l'âge qu'il avait quand son propre père est mort. Est-ce que vous seriez d'accord?).

Ces contributions profanes à l'examen de la personnalité peuvent venir contrebalancer des expertises, voire s'y opposer. Un président le souligne par exemple lors d'un échange avec un enquêteur de personnalité: Ce que vous dites-là est intéressant parce qu'il y a une psychologue qui nous dit exactement le contraire, que c'est quelqu'un de très impulsif. L'enquêteur répond, justifie longuement son propos et conclut: mais après, je ne suis pas expert. Pour un autre accusé, question du président: vous concluez sur une personne réservée, qui s'inscrit dans les normes sociales, respectueux. Est-ce que c'est pas un peu lisse? Et l'enquêteur de défendre son point de vue.

Autrement dit, si l'on admet, ce que nos observations montrent amplement, que les expertises n'ont pas seulement pour objet d'évaluer la présence de troubles mentaux, la dangerosité ou la réadaptabilité mais qu'elles doivent éclairer le jury sur la personnalité des accusés, et qu'à ce titre elles appartiennent à un ensemble comprenant également les enquêtes de personnalité et d'autres contributions profanes, la manière dont est organisé cet examen lors du déroulement de l'audience joue un rôle primordial en donnant plus ou moins de force aux différents propos. La contribution des différents développements «psy» au jugement repose moins sur une hiérarchie a priori des légitimités que sur la contingence du déroulement de l'audience.

\section{Le président d'assises et les évaluations "psy»}

Une troisième série d'éléments vient encore perturber le schéma initialement évoqué: le président décide de donner plus ou moins d'ampleur à cette étude de la personnalité, selon l'importance ou le type d'affaires, la conception qu'il a des débats d'assises et de la place que doivent y prendre les expertises $"$ psy ${ }^{12}$. Il n'est pas un simple exécutant du droit mais un interprète, il bénéficie d'une autonomie très large de son action. Le "pouvoir discrétionnaire» dont il est investi lui permet de «prendre toutes mesures qu'il croit utiles pour découvrir la vérité» (art. 310 du code de procédure pénale). C'est également le président qui a «la police de l'audience et la direction des débats» (art. 309 du CPP).

Il est possible de discerner clairement un intérêt plus ou moins grand des présidents, au travers de l'examen de la personnalité, pour la question de la responsabilité subjective

12 Un président nous a par exemple expliqué qu'il juge opportun de faire intervenir les experts à la fin du procès dans la mesure où l'expert est ainsi susceptible de répondre à l'ensemble des questions soulevées tout au long de l'audience. D'autres manifestent un souci de gestion du bon déroulement du procès. 
à laquelle la défense sociale s'est attachée. Le président peut considérer comme négligeables les expertises au vu des affaires. Lors d'un procès en appel contre six auteurs d'incendies volontaires et dégradations, le président va vite sur l'examen des personnalités: les experts sont absents et leurs conclusions ont été partiellement lues, les enquêteurs de personnalité s'enchaînent et l'on finit par ne plus savoir de qui ils parlent: soit que les accusés ne se lèvent pas à leur tour, soit qu'un même enquêteur évoque plusieurs jeunes à la suite (Président: Tant que Mme Raymond est là, on peut peut-être l'entendre sur la personnalité de M. Zémour). Il s'agit ici pour lui de gérer la durée du procès.

Le président guide les débats, ce qui signifie qu'il donne la parole aux experts, puis les interroge, reprend leurs discours et enfin distribue la parole aux parties. Le style de cette conduite importe puisque, par exemple, il peut proposer aux parties d'intervenir de manière à simplement respecter le droit sans attendre véritablement d'interventions: $M$. l'avocat général, une question? La défense, quelque chose à ajouter? Il peut donner la parole à l'accusé sous des formes différentes, selon des degrés de directivité plus ou moins grands, depuis la question ouverte (Que pensez-vous de ce que vient de dire l'expert?) aux questions plus ou moins orientées et directives (Que pensez-vous de ce que vient de dire l'expert de l'absence de votre mère?) ou qui n'appellent pas de réponse (Le décès de ce père vous a durablement marqué).

Intervenant à la suite des témoins, des auxiliaires de justice, il peut poser des questions d'informations, d'éclaircissement, soulever des imprécisions, des contradictions et pour cela interroger les experts sur ce qu'ils viennent de dire ou sur ce qu'ils ont écrit, ou sur ce que d'autres ont dit ou écrit, proposant là encore plus ou moins une interprétation. Il peut surenchérir sur tel ou tel élément en puisant dans le dossier.

Par sa manière de conduire les débats, de distribuer la parole, de poser des questions mais aussi de lier des pièces du dossier entre elles, de piocher dans des procès-verbaux, des expertises, des rapports d'enquête, le président peut donner à entendre une version des faits, il donne de toutes façons du sens, il propose implicitement par ses rapprochements des liens de causalité entre les faits, entre la personnalité et les faits.

Dans un procès pour braquage (nié par l'accusé), le président considère manifestement que la violence de l'accusé est patente et que cette personnalité rend possible sinon probable sa participation aux faits. Dès la première demi-journée, après l'interrogatoire de curriculum, il développe une première fois son idée: Pour éclairer ce tempérament violent dont on vous qualifie (...) et enchaîne en citant des extraits de procès-verbaux de témoins. Se tournant vers les jurés: C'est important à noter parce que l'inculpé est accusé de vols comportant une forte dimension de violence. Quelques minutes plus tard, se tournant vers l'accusé: C'est une violence que vous expliquez par la violence que vous faisait subir votre papa... L'accusé répond: Ça n'a rien à voir avec le dossier. Le président poursuit sans s'arrêter à l'objection et termine son intervention par: Vous êtes décrit comme toxicomane et violent. Voilà. Le lendemain, après l'intervention du psychologue qui n'a pourtant pas évoqué cet élément:

- Président: Et la violence qu'est-ce que vous pouvez nous en dire?

- Expert psychologue: Dès que les affects sont concernés, que son narcissisme est convoqué, il peut effectivement avoir des comportements violents.

- Président: Ces éléments psychologiques sont importants pour nous aider à comprendre les faits. Il y a dans les faits reprochés des comportements violents que l'on 
peut retrouver dans la personnalité. Alors je ne dis pas que ça fait de lui un coupable désigné...

- Expert psychologue: Oui quand il y a frustration il peut avoir des actes de violence ou d'impulsivité.

Dans cette séquence, le président introduit le thème de la violence que le psychologue n'avait pas mentionné. Puis il explicite sa démarche, le sens de ses questions: l'accusé niant les faits, il s'agit de savoir s'ils sont congruents avec une personnalité donc s'il peut être coupable. En formulant sa proposition sur la culpabilité de l'accusé sous la forme négative et en laissant sa phrase en suspens, il signale à l'ensemble du jury et de la cour qu'il ne s'agit que d'une suggestion. Enfin, par deux fois, sollicité par le président, l'expert répond par l'affirmative: l'accusé peut, dans certaines conditions, se montrer violent.

Plus globalement, il est fréquent que l'expert infléchisse son jugement suite aux questions ou propositions interprétatives du président. Dans l'exemple qui suit, pointant deux affirmations qu'elle tient pour opposées dans l'exposé de l'expert, la présidente suggère que la réadaptabilité pourrait peut-être être réévaluée. Si dans un premier temps l'expert se montre relativement pessimiste quant à la réadaptabilité de l'accusé, concluant que la réadaptabilité est plus que compromise, la présidente mobilise un autre élément présent dans son exposé:

- Expert: Donc on a une espèce de dérive délinquantielle avec une sorte de crescendo dans le passage à l'acte et qui se termine aux assises.

- Présidente: Alors c'est vrai que dans son parcours on peut supposer que les faits commis aujourd'hui apparaissent un peu plus euh, c'est que des suppositions, mais ce que vous avez dit concernant le fait qu'on peut compter sur le vieillissement, que c'est un âge où on commence à se calmer... est-ce qu'on est ici dans ce processus ou pas?

- Expert: Je parle des qualifications pénales. Après, le contenu, c'est une autre affaire effectivement, mais moi je note qu'on était en correctionnelle et qu'on passe ensuite deux fois aux assises, y'a quand même eu un crescendo, quel que soit le conte$n u$ des faits qui sont jugés. Par contre, je crois que la dynamique que j'évoquais tout à l'heure, de tassement avec l'âge, on peut effectivement s'attendre à une réduction de son potentiel criminogène et délinquantiel avec l'âge. Maintenant vous savez, ça c'est une estimation globale...

L'expert clôt donc la séquence sur ce thème en accédant à la proposition qui lui est faite: «on peut effectivement». Le pronostic de réadaptabilité, comme les autres conclusions inscrites dans le rapport d'expertise n'est pas figé, il est retravaillé dans les échanges. Il est, non pas contesté sur la base d'autres expertises, mais nuancé sur la base de propositions alternatives, profanes, en particulier des présidents.

Tel président ne se privera pas de résumer ce qui vient d'être dit par un psychiatre (Vous avez bien décrit si j'ai bien compris quelque chose qui ressemble à une névrose), l'intérêt de tel test projectif (Mesdames et Messieurs les jurés, ces tests peuvent paraître farfelus mais tous les psychologues utilisent ça depuis le XIX ${ }^{e}$ siècle [la psychologue donne des éléments sur l'histoire des tests et leur validation par la communauté scientifique]. Voilà, 
il est important que vous disiez tout cela pour bien montrer que c'est très sérieux et que ce n'est pas du charlatanisme) ou la définition d'une catégorie nosographique. Les termes de «pervers» ou de «sociopathes » peuvent, selon le contexte de leur énonciation, prendre un sens technique, être plus ou moins explicités ou, à l'inverse, se charger de la condamnation morale qu'ils contiennent dans leur usage vulgarisé. Le président joue alors un rôle essentiel dans le sens pris par les mots, soit en n'intervenant pas, soit en demandant à l'expert de s'expliquer ${ }^{13}$, soit encore en proposant lui-même une interprétation.

Le président à l'expert psychologue: Dans votre rapport vous avez écrit: «traits psychopathiques» [Se tournant vers les jurés]: Je le dis, attention, on n'est pas au café du commerce ici, le psychopathe ce n'est pas un malade mental, c'est quelqu'un qui ne veut [accentue] pas accepter les règles de la société. [Se tournant à nouveau vers l'expert]: Vous avez écrit que le délit s'inscrit dans la poursuite des besoins immédiats. Donc le pronostic de réadaptation reste incertain... [invitation à répondre].

Dans un autre procès, pour viol, l'expert psychiatre fait un compte rendu très succinct de son expertise (trois minutes). Après des questions de l'avocat général, de la partie civile et de la défense, le premier réinterroge l'expert:

- Avocat général: La générosité compulsive, ce n'est pas une forme de perversité?

- Expert: Non pas au plan psychiatrique, mais sinon, oui. Quelque part c'est comme dans une relation avec un objet.

- Avocat général: J'ai assisté à un colloque, on parlait de la dépersonnalisation. L'autre n'existe pas en tant que tel...

- Expert: Ce n'est pas un pervers au plan psychiatrique, il faut voir avec l'autre expert qui l'a vu tranquillement.

- Intervention de l'avocat de la défense: Rien dans les rapports psychologiques et psychiatriques ne parle de perversité.

Le débat avec l'expert se clôt ainsi, le président n'est pas intervenu sur l'expertise. Les parties manipulent les sens moral versus technique du terme "pervers» et l'expert ne rejette pas l'interprétation vulgaire de la notion qui lui est proposée.

Dans un troisième cas - procès pour viol incestueux - le président demande à l'expert d'expliciter la formule «absence de déviance perverse» qu'il vient d'utiliser dans l'échange:

- Président: Il y a une planche sexuelle, qu'est-ce qu'elle permet de savoir?

- Expert: Il y a des personnes qui ne peuvent pas répondre et cela dénote une inhibition grave. Ici, il y a une réponse banale et adaptée. Il y a une absence de déviation perverse de la vie sexuelle.

- Président: C'est quoi le fonctionnement pervers?

- Expert: C'est une recherche de satisfaction qui ne passe pas dans une sexualité différenciée, c'est-à-dire une absence de partage avec une place spéciale, peu valorisée au partenaire. Par exemple les pédophiles, les masos.

13 Dans un entretien avec un président celui-ci nous déclare: si on dit pervers ou pédophile, on est sûr que la peine va augmenter. Quand l'expert prononce ces mots, je les reprends, je leur demande d'expliquer. 


\section{Dégager la responsabilité morale de l'accusé}

La psychiatrie a pris très progressivement au cours de l'histoire une place croissante au tribunal. Dans le code pénal de 1810 la responsabilité est objective et calculée d'après la nature de l'infraction et non d'après la personnalité de son auteur qui est soit responsable, soit irresponsable de manière dichotomique. La circulaire Chaumié de 1905 introduit une première nuance avec la notion de «responsabilité atténuée», en invitant les psychiatres à rechercher dans quelle mesure l'accusé peut révéler « des anomalies physiques, psychiques ou mentales » ne relevant pas de l'aliénation mentale au sens de l'article $64 \mathrm{du}$ code pénal de 1810. Quant au code de 1994 dans son article 122-1, alinéa 2, il évoque une «atténuation de la responsabilité». Mais les acteurs ne sont pas, dans les procès observés ici, à la recherche de la «folie partielle » ou de la «responsabilité atténuée»: les acteurs du procès cherchent, avec les experts, à cerner l'élément moral du crime c'est-à-dire les capacités cognitives, l'engagement de la volonté, les éléments subjectifs de la personnalité.

Lélément moral consiste en l'intention de commettre le crime. C'est ce niveau d'intention que les échanges autour de la personnalité de l'accusé ont entre autres pour but d'éclairer. Loin d'être une grande catégorie que l'on pourrait essentialiser, une notion philosophique indépendante du contexte institutionnel de sa mobilisation, [l'intention] la responsabilité apparaît comme le résultat d'interactions s'insérant dans le contexte institutionnel judiciaire ${ }^{14}$.

Nous avons discerné trois grandes thématiques explorées par la Cour au travers de l'examen de la personnalité et des caractéristiques "psy» de l'accusé: l'élément moral proprement dit, la congruence entre la personnalité et les faits et, enfin, la réflexivité de l'accusé.

\section{Savoir si l'élément moral du crime est constitué}

\section{Les confusions dans le discours de M. Éloi : un problème «psy»?}

M. Éloi est jugé en appel pour viols sur personne vulnérable (handicapée mentale). Âgé de 34 ans au moment du procès, il était intérimaire en maçonnerie. Il est originaire d'Outre mer. Les faits ont été commis neuf ans plus tôt. Durant la lecture de l'arrêt de renvoi on apprend que Mr Éloi aurait contesté sa culpabilité, considérant qu'il n'avait pas violenté la jeune femme, qu'elle n'avait pas manifesté d'opposition: Interpellé, il admettait les faits mais déclarait qu'il ne l'avait pas violée. Il s'agit d'un procès en appel. En première instance, un an plus tôt, Mr Éloi avait été déclaré coupable et condamné à quinze ans de détention.

Le procès débute à $9 \mathrm{~h} 30$ et se termine, avant le délibéré, à $20 \mathrm{~h} 15$. Les deux premières heures sont consacrées à un interrogatoire du président sur la personnalité de l'accusé, sa biographie, suivies de quelques questions des parties civiles, du ministère public. A posterio$r i$, on peut dire que la journée a été centrée sur la personnalité au sens des capacités cognitives de l'inculpé et de sa volition, ce que le président nommera «l'élément moral» de l'acte. Une première séquence d'échanges, plutôt le matin, vise à cerner si l'accusé a des problèmes

14 Dupret (2001b, 347). Et encore: La personne consciente et intentionnelle, loin d'être une catégorie abstraite et inaccessible, est constituée publiquement, grâce au déploiement méthodique de ressources publiques, c'est-à-dire linguistiques, dans l'interaction sociale, Dupret (2001a, 453). 
de mémoire, s'il est parfaitement conscient de ses actes, s'il réussit à les situer dans le temps, dans leur enchaînement. La seconde, l'après-midi concerne la conscience d'avoir commis un viol. On verra la place exacte de l'expertise psychiatrique dans ces deux séquences.

10 h 15. Le président: M. Éloi, je vais vous demander de vous présenter. Parlez-nous de vos vingt-cinq premières années.

M. Éloi raconte et le président poursuit son interrogatoire:

- Président: Quel âge avait votre père quand il est décédé?

- M. Éloi: 70 ans

- Président: Dans l'enquête de personnalité, il est dit 60 ans, le psychologue a noté 75 , il semble que vous ayez du mal à vous repérer dans le temps. Est-ce que vous savez quand sont nés vos père et mère?..

Dans une longue séquence d'une heure, le président fait raconter à l'inculpé sa vie. Il produit une présentation des conditions socioculturelles dans lesquelles l'inculpé a vécu, et cherche à donner à entendre aux jurés autant qu'à cerner pour lui-même les capacités intellectuelles et cognitives de l'accusé. Progressivement, cette quête se focalise sur ce qui pourrait constituer un indice: une mémoire défectueuse. Le président explorera d'ailleurs une piste: le traumatisme consécutif à un accident de la circulation a-t-il pu entamer les capacités cognitives de l'accusé? Toute cette séquence se déroule en l'absence des experts ${ }^{15}$. Le président s'appuie essentiellement sur l'enquête de personnalité - il citera l'enquêteur de personnalité citant un employeur: Il est un peu simplet mais il travaille bien - et les témoignages - Mme Gride dit de vous, "c'était un fada, il n'était pas méchant» ainsi que sur un interrogatoire serré. Le président ne cherche pas seulement à évaluer des capacités, il les manifeste pour les jurés en donnant longuement la parole à l'accusé. Il ne propose pas de conclusion.

La question de la mémoire reviendra de manière centrale l'après-midi lors de l'examen des faits: une longue série de questions auront pour but tout autant de déterminer l'ordre des faits - comment se sont déroulées les rencontres, à quel viol correspond tel ou tel événement... - que d'exhiber les défaillances cognitives.

Les interventions de l'avocat de la partie civile, de l'avocat général portent sur la personnalité avec les questions suivantes: Il se décrit comme un «tigre solitaire; est-ce qu'il confirme ce propos? Les témoins et enquêtés disent que vous n'êtes pas très intelligent, simplet, est-ce que vous êtes d'accord? Vous dites que vous avez été jusqu'en terminale, est-ce que vous ne rêvez pas un peu? Dans le contexte et prononcée par l'avocat de la partie civile, la question vise à opposer, à une présentation de l'accusé comme limité intellectuellement - mais pas méchant - la figure d'un individu qui ment, ajoutant d'ailleurs pour compléter le portrait: Certains disent que vous êtes susceptible, est-ce vrai?

Une seconde phase de cette séquence relative aux capacités cognitives de l'accusé débute à $17 \mathrm{~h} 55$, avec l'audition des experts. En l'absence du psychiatre, c'est le psychologue qui, regardant épisodiquement ses notes ou son expertise déclare que il est lucide, il a des capacités cognitives normales, il perçoit la chronologie, sans distorsion.

15 On remarquera toutefois - c'est un avocat qui le déclare - que l'enquête de personnalité a été réalisée par une psychologue. 
Alors qui est cet homme? Pas de troubles intellectuels pour faire simple, fonctionne au plan cognitif à peu près correctement. Reste la question de conséquences de son accident... Le psychologue fait référence à des épreuves qu'il a fait passer, à des tests - en particulier projectifs - à des mesures et à un savoir général sur la structuration de la personnalité. Il apporte des éléments et des réponses très précis sur la mémoire. Deux questions lui sont d'ailleurs posées en ce sens. L'avocat de la défense revient plus globalement sur les capacités intellectuelles limitées, l'expert répondant en distinguant les différentes dimensions de l'intelligence.

\section{Dans quelle mesure M. Éloi savait-il qu'il commettait un viol?}

Comme l'ont montré différents auteurs dans des analyses sur le droit en action, une part du travail juridique consiste, pour les acteurs, à confronter des faits aux catégories juridiques - et ce faisant, leur donner une réalité. Comment font les acteurs pour décider s'il s'agit d'un viol? (Matoesian, 1997; Dingwall, 2000; Dupret, 2001a, b). Ils s'appuient en partie, comme nous avons pu le constater, sur un examen de la personnalité, avec ou sans les experts.

Cette seconde séquence débute l'après-midi avec cette question du président: $M$. Éloi, qu'est-ce que c'est, pour vous, un viol? À quoi l'accusé répond: Le viol, c'est quand on la frappe.

L'accusé avait bien l'intention d'avoir une relation sexuelle avec la victime, il ne s'en cache pas. Mais dans quelle mesure avait-il conscience qu'elle s'y opposait, qu'il la contraignait, sachant qu'elle n'était pas forcément capable - étant handicapée mentale - de manifester clairement cette opposition? La difficulté se double d'une interrogation sur la culture de l'accusé c'est-à-dire sa conception des relations hommes-femmes, particulièrement sur le plan sexuel. Le terme «culture» étant entendu ici comme le résultat d'une socialisation sur un territoire, au sein d'une société très différente de la métropole française ainsi qu'au sein d'une famille à la composition bien spécifique. Dans son interrogatoire de personnalité, le président va revenir à plusieurs reprises sur la vie sentimentale et sexuelle de l'accusé:

- Président: On va revenir maintenant sur votre vie affective. À quel âge avez-vous eu vos premières relations sexuelles?

- M. Éloi: À onze ans.

- Président: Pourquoi toujours avec des femmes mûres? Parce que l'expert a noté que vous étiez agophile...

Il l'interroge sur une relation qu'il aurait eue avec une femme handicapée, ce dont il se défend en expliquant qu'elle suivait simplement un traitement. Il cherche à caractériser ainsi la sexualité de l'accusé afin d'établir si l'acte pour lequel il a été condamné correspond à une attirance particulière pour ce genre de personne. À aucun moment le président ne donne l'impression de défendre une conception normative de la sexualité.

Toujours pour caractériser cette sexualité, le président pose une série de questions sur la manière dont il fait connaissance avec des femmes, les aborde, leur propose des relations sexuelles, sur sa «technique de drague ». Pour rencontrer les personnes avec qui vous avez des relations, vous faites comment en général? Puis des questions sur sa conception 
de la femme, en particulier en tentant de relier celle-ci à une sous-culture ou une socialisation particulière. Il s'agit de savoir si pour lui il est normal d'avoir des relations sexuelles avec une femme sans s'assurer de son consentement:

- Président: Comment vous voyez la femme M. Éloi?

- M. Éloi: La femme je la vois la santé, le respect, subvenir aux besoins des enfants.

Des questions visent à donner à entendre aux jurés si le handicap de la victime était manifeste ou si l'accusé pouvait ne pas s'en être aperçu et si dans son état elle pouvait avoir un intérêt pour la sexualité. Sur ce point, un psychiatre qui a expertisé la victime est entendu et décrit pour les jurés le comportement, l'état, l'apparence habituelle des personnes atteintes de cette déficience. Il soutient qu'aucun doute n'est possible. Les parties prolongent cette interrogation par leurs questions à l'expert de la victime. M. Éloi pouvaitil se méprendre sur l'état de la plaignante? Les questions au psychologue appelé ensuite à propos de l'accusé tournent encore essentiellement sur cette question: Avec les capacités que vous nous avez décrit, pouvait-il percevoir l'absence de consentement? Les questions à cet expert portent, d'une part sur cette capacité de l'accusé à comprendre le sens de ses actes - le psychologue répondant de manière technique (épreuves, tests), et sur ce qu'apprend l'examen de la sexualité de l'accusé.

S'agissait-il d'un viol? En l'absence de preuves matérielles, de témoins des faits, en raison de la possible incapacité de la victime à exprimer clairement un refus, le président a éclairé la cour sur la personnalité de l'accusé, il a visibilisé une conception des relations hommes-femmes, et le psychologue aura, en outre présenté une évaluation de la compatibilité de la personnalité et des faits reprochés. Dans ce procès, le président place le jury dans la situation de devoir décider s'il s'agissait d'un viol en tenant compte de la conscience que pouvait avoir l'accusé de ce qu'est un viol.

\section{Faire correspondre des faits et une personnalité}

Un second grand thème est exploré avec l'examen de la personnalité et participe cette fois à juger de la culpabilité: la congruence de la personnalité et des faits reprochés ${ }^{16}$.

L'exploration de la personnalité des accusés par les experts est très directement liée aux faits qui leur sont reprochés, qu'ils reconnaissent ou non les faits ou, comme il est d'usage de le relever dans les rapports d'expertises, «sans préjuger de la culpabilité». On ne retient pas les mêmes éléments de l'enfance, des tests, des témoignages s'il s'agit de vols avec violence, d'un viol, de trafic de stupéfiants, d'abus de biens sociaux.

Les professionnels présents au procès savent que, pour les jurés, l'affaire cohérente au niveau du sens peut être adéquatement jugée comme étant ce qui s'est réellement passé. Si l'interprétation est plausible, c'est alors ce qui s'est passé ${ }^{17}$.

16 B. Dupret rappelle que ce jugement de congruence a été mis en lumière par les ethnométhodologues: L'étude de Harvey Sacks sur l'évaluation par la police du caractère moral des prévenus permet de saisir comment tout le travail d'évaluation des faits, objets et personnes opère à partir de la typicalité de situations "routinisées", tandis que l'article de D. Sudnow sur les crimes normaux s'attache à montrer que cette normalité est construite à partir des caractéristiques typiques prêtées à ces situations et attendues d'elles (2001b, 345).

17 Garfinkel, 2007, 190. 
Dans un procès pour viol, le président interroge l'expert psychologue qui a examiné l'accusé en lui posant d'abord des questions sur sa biographie, puis des questions plus générales sur les tests. Il conclut alors ses questions par la séquence suivante:

- Président: Dans ce cas, c'est le profil psychologique du père incestueux?

- Expert psychologue: Oui, on a des statistiques. Il n'y a pas de causes sociales. Il a les traits du profil du père incestueux: ambivalence affective - très chaleureux et en même temps autoritaire et possessif - qui permet d'exercer une emprise sur la personne qui va être victime. Il a des difficultés à tenir le rôle du père. Au lieu de référents extérieurs à la loi du père, il y a un fonctionnement égocentrique. Mais il a aussi des difficultés à s'occuper d'un bébé, comme il me le dit. On est plus dans une relation banalisée, plutôt que parentale.

[Pas de question de la partie civile]

- Avocat Général: Sans préjuger de la culpabilité de l'accusé, la typologie du père incestueux correspond à celui-ci sur tous les points?

- Expert psychologue: Oui mais on ne peut préjuger de la culpabilité, c'est plus pour éclairer le contexte oedipien.

- Avocat général: Ça crédibilise les propos de la victime?

- Expert psychologue: Elle décrit le même fonctionnement et elle s'en plaint. Sur les faits je n'ai pas d'éléments pour confirmer la version de l'un ou de l'autre.

- Avocat général: Les deux sont d'accord sur le contexte.

- Expert psychologue: Oui, le contexte a pu favoriser les débordements.

À travers ces échanges on voit que ce qui est cherché par les protagonistes, c'est une cohérence entre les faits et la personnalité («le profil psychologique»). L'expert dit ne pas se prononcer sur les faits, mais il indique que le savoir psychiatrique le plus objectif (des «statistiques ») rend très probable les faits au vu de la personnalité. Dans le même temps, afin de n'être pas accusé de ne pas se tenir à sa place ${ }^{18}$, il prévient l'objection: sans préjuger de la culpabilité de M. X.

Cette recherche du «typique» dans la personnalité qui rend l'imputation des faits très vraisemblable est fréquente. Deux autres exemples:

- Avocat de partie civile: C'est le premier rapport sexuel qui déclenche la dénonciation des faits, c'est fréquent chez les victimes?

- Expert psychiatre: Oui, c'est quand la victime a l'occasion de sortir de cet univers. Ce sont des éléments que l'on retrouve systématiquement dans les cas incestueux.

- Avocat général: Ce sentiment de culpabilité, il est typique des victimes d'incestes? - Expert psychiatre: Oui et le silence de la victime s'explique par ce sentiment de culpabilité.

- Avocat général: Il y a une ambiguité: elle dénonce les faits et ne veut pas de punition, c'est normal? Parce qu'il ne faudrait pas croire qu'il ne s'est rien passé.

- Expert psychiatre: Oui, c'est normal.

18 Expression utilisée par un magistrat lors d'un entretien à propos des experts qui prennent parti, ne s'en tiennent pas à ce qui relève de leurs compétences. 
L'utilisation des termes comme «typique» ou encore «systématique», «fréquent», «normal» objective l'affirmation en la rapportant au cas général connu et décrit par la science. Ce cas de figure doit être nettement distingué de celui dans lequel l'expert évoque une relation de causalité, une étiologie de l'acte criminel. Il ne s'agit pas ici d'expliquer que telle structure psychique est la cause de tel passage à l'acte mais d'opérer un simple rapprochement, une juxtaposition sans lien affirmé entre des faits allégués, ils peuvent être niés, et des traits de personnalité. En outre, il est remarquable que ce travail de mise en cohérence soit très fréquemment sollicité par les acteurs de la sphère judiciaire.

\section{Savoir si l'accusé est capable de travailler sur luii-même}

Dans une troisième et dernier cas de figure, les développements "psy» participent à la décision relative au quantum de peine au travers de la question de la possibilité de sortir de détention plus ou moins rapidement, sans danger pour la société. Pour les magistrats comme pour les experts, la possibilité de sortir de prison si l'on est en détention préventive, si l'on est jugé en appel ou si l'on risque d'être condamné à une peine de prison est liée à la possibilité de l'adaptation à l'ordre normal des choses, elle-même conditionnée à une norme : la capacité à faire un travail sur soi.

On pourrait faire l'hypothèse que ce sujet que l'audience promeut, qui doit se montrer acteur de son propre parcours de justiciable, faire un travail sur soi pour produire sa vie participe de cette nouvelle forme de gouvernementalité caractéristique de la dernière modernité libérale ${ }^{19}$.

La littérature sur l'expertise psychiatrique a relevé que l'engagement dans des soins «psy» en détention pouvait faire l'objet de la part des acteurs d'une instrumentalisation en vue des aménagements de peine, voire des conditions de détention. Ce que montrent les audiences, c'est que l'engagement soit dans des soins «psy» - et l'authenticité de cet engagement - soit dans un travail personnel sur soi est également pris en compte pour estimer sinon le quantum de peine, du moins le temps d'un cheminement à opérer pour prévenir la récidive. C'est en tout cas ce que les présidents, les parties engagent le jury à faire en lui soumettant des éléments de jugement sur ce point. Alors que dans le cas de figure précédent les «psy» devaient permettre de juger de la culpabilité au sens de: «son profil rend-il sa culpabilité possible ou probable», les acteurs font ici tout autre chose.

Dans cet exemple, après que le psychiatre en ait terminé, le président interroge l'accusé:

- Président: Qu'est-ce que vous avez compris de ce qu'a dit le psychiatre?

- Accusé: Qu'il y avait un lien entre mon enfance et les actes de délinquance.

- Président: Et sur la réinsertion?

- Accusé: J'ai entamé un travail psychologique sur moi en incarcération.

- Président: Vous avez compris que vous êtes à la croisée des chemins? On doit prendre en compte les intérêts de la société, c'est notre mission. Vous je voudrais savoir où vous vous situez...

- Accusé: Je fais un travail sur moi. Avant j’avais jamais fait de suivi.

19 À la suite de Garland (2001) pour le crime, de Rose (1989) pour les troubles mentaux, de Vrancken, Macquet (2006) pour la question sociale. 
Dans leurs expertises, les experts cherchent, de manière récurrente et transversale aux missions, à évaluer si l'inculpé fait retour sur lui-même, s'il donne du sens à son histoire, s'il se distancie de ses actes. Les formes rhétoriques très fréquentes que sont: il dit que..., il pense que..., il considère que... n'ont donc pas seulement pour but de mettre la vérité des affirmations entre parenthèses ou, dans d'autres contextes d'énonciation, de les mettre en doute. Typiquement, l'énoncé «psy»: Il se situe exclusivement au niveau du discours signifie dans ce contexte l'absence de réflexivité de l'accusé. Le sujet fait-il un retour réflexif sur lui-même? Mentalise-t-il ses actes, ses affects ? Élabore-t-il des contenus psychiques? Autant d'indications que l'accusé, le sujet est en train ou capable d'opérer des changements profonds dans sa personnalité. Et donc qu'il pourrait ne pas réitérer et se réinsérer.

Ce sont ces opérationnalisations de théories psychodynamiques qui sont mises en œuvre lors du procès. Non seulement par les «psy» mais à la demande de la cour:

- Président: Quelle est la part de responsabilité de l'individu dans sa destinée?

- Psychologue ${ }^{20}$ : Donc chaque individu va réagir différemment. Et l'enfant porte une responsabilité individuelle. C'est un freudisme mal digéré qui considère que l'enfant n'est pas acteur. C'est entièrement faux. L'enfant est acteur très rapidement. Soit il rentre dans un travail de maturation par rapport à la frustration, soit il est dans le refus. Pour Mr Exit, c'est vrai que quand son père disparaît, il n'y a pas de sens donné à cette disparition. Il n'a pas pu me dire pourquoi son père avait disparu. Il n'a pas pu mentaliser, il n'y a pas de représentation psychique. Le travail de deuil ne s'est pas bien fait. Alors le travail avec le psychologue, qu'il a entamé en détention, ça peut le faire bouger. La responsabilité de la réaction elle y est: il n'est pas dans l'ordre de l'aliénation. Il doit se reconnaître responsable de ses actes pour avancer. Le sujet juridique est sommé de se faire sujet analytique: "je veux me connaître enfin moi-même». En se connaissant, il se libère de ses pulsions, de ses affects ».

Autre procès :

- Président: Qu'est-ce qu'on peut penser du fait qu'il se soit marié, ça c'est une chose, mais qu'est-ce qu'on peut aussi penser du fait qu'il nous dise: "avant, quand jétais incarcéré, y'a que moi que ça gênait, donc je gère ça, voilà... maintenant je rends la vie impossible à... euh et j'ai quelqu'un à qui je rends la vie impossible, et ça, je veux plus le revivre». Il nous dit que, pour lui, c'est un élément important et qui peut nous laisser penser qu'il ne va pas récidiver... Qu'est-ce qu'on peut en penser?

- Expert psychiatre: Moi ce que je sais c'est que lorsque je l'ai vu, il était dans une logique plutôt de loup solitaire, il disait qu'il gardait sa liberté qu'il souhaitait pas d'accroches affectives. Il se peut effectivement qu'il y ait des choses qui se soient élaborées dans sa tête, et qu'effectivement ses engagements affectifs lui permettent d'élaborer son existence de manière différente. De là à dire que puisqu'il s'est marié il est moins dangereux alors là... j'irais pas jusque-là.

20 Il débute par une réponse générale que nous n'avons pu retranscrire sur les étapes de l'autonomisation psychique, les réactions et étapes de l'inconscient face aux situations de perte. 
On voit ici que ce n'est pas l'élément matériel - s'être marié ou non - qui importe, mais bien ce que dit l'accusé du fait de s'être marié, qu'il se perçoit différemment et considère désormais la portée de ses actes pour les autres. Avec les experts, on examine au procès les propos que l'accusé tient sur ses actes et sur lui-même, ses processus de pensée, ce qu'ils indiquent de sa capacité d'élaboration mentale, de subjectivation.

\section{Cheine a-t-il véritablement entamé un travail sur soi ?: l'expert poussé à se prononcer}

Dans un procès, lors de la lecture de l'acte de mise en accusation, il est fait allusion au fait qu'après sa première incarcération, l'accusé «a voulu se soigner» en allant voir un psychiatre mais que cette tentative avait échoué. Il avait consulté un médecin pour ses problèmes de violence mais déclarait ne pas avoir donné suite. Il déclarait ne pas pouvoir se contrôler.

Cet élément sera discuté plusieurs fois au cours de l'audience, en particulier au travers d'échanges avec les experts, pour déterminer s'il avait réellement tenté de se soigner. Ce point est capital dans la mesure où il met en balance deux figures de l'accusé: l'accusé qui a conscience de ses difficultés, qui tente d'y remédier - comme en d'autres cas on doit faire la preuve qu'on a cherché à s'amender - mais qui en a été empêché par ses troubles ou sa personnalité même, versus un accusé qui n'a pas véritablement pris acte du caractère criminel et odieux de ses actes, qui n'a pas voulu se donner les moyens de cesser ses violences. L'accusé est-il responsable de ses actes s'il sait qu'il a des impulsions violentes (versant déterministe) et qu'il ne se soigne pas (versant liberté de l'individu)? Mais aussi, comment ne pas le placer et le maintenir en détention si, ayant besoin de soins sous peine d'être dangereux, il ne s'y résout pas?

Dans ce procès, le psychiatre intervient en premier et soulève rapidement ce qui lui semble le problème crucial: La question qui se pose c'est quand même celle de ce qu'il peut devenir. Il doit être sevré des drogues, il doit s'engager dans un travail en profondeur. Il me paraît indispensable qu'il puisse travailler sur son histoire. Si ces deux conditions sont remplies, il a des capacités de réinsertion. Dès lors, le décor est planté et la question sera abondamment discutée: veut-il vraiment se soigner?

Les premières questions au psychiatre visent à évaluer l'ampleur de cette impulsion à la violence. Puis c'est au tour de l'avocat général:

- Avocat général: Ce qui me paraît notable, c'est qu'il est conscient de son problème de violence, qu'il a tenté de se faire soigner mais que sa compagne l'en a empêché... - Expert: Je ne pense pas qu'il y a ait eu vraiment un désir d'entrer dans les soins. - Avocat général: Compte tenu de sa personnalité est-ce que sa compagne aurait pu l'empêcher de se soigner?

- Expert: Je ne pense pas que ça ait une influence, c'est d'abord à lui de décider. - Avocat général: Parce que ce qu'il dit, c'est que sa compagne l'en aurait empêché.

Vient ensuite le tour de l'avocat de la défense:

- Avocat de la défense: Qu'aurait-il dû faire pour confirmer son désir de soins?

- Expert: Oh lala, je ne sais pas... À partir de là qu'est-ce qu'il faudrait faire je ne sais pas... 
- Avocat de la défense: On n'aurait pas permis à M. Cheine d'entrer dans les soins, on s'est contenté de lui donner des médicaments. Est-ce qu'il n'aurait pas fallu lui proposer une autre prise en charge, un vrai suivi?

- Expert: Je le répète parce que vous me le faites répéter, oui il aurait fallu.

- Avocat de la défense: Est-ce que sa conjointe n'aurait pas pu l'aider à se soigner?

- Expert: Je n'ai aucun avis là-dessus, je ne l'ai pas rencontrée.

Les parties poussent le psychiatre à se positionner quant à la qualité de l'engagement de l'accusé. S'il le fait à un moment - Je ne pense pas qu'il y ait eu vraiment un désir d'entrer dans les soins - il se montre ensuite agacé du jeu qu'on veut lui faire jouer. Il affirme son incompétence pour juger si une quelconque situation a pu l'en empêcher.

À la fin de l'audition de l'expert psychiatre, le président réinterroge M. Cheine sur sa capacité à se contrôler. À quoi celui-ci répond: je suis suivi depuis trois ans que je suis en prison. Et le président de déclarer, à l'adresse du psychiatre: Se pose la question du lieu contenant. Autrement dit, avec cet énoncé, une invitation faite aux jurés à se demander : s'il sort, puisqu'il est connu de tous que ces lieux n'existent probablement pas dans le secteur sanitaire, ne représente-t-il pas un danger pour la société?

Les échanges sur ce point ne sont pas terminés puisque le médecin qui a prescrit les médicaments à l'accusé quand il est venu le voir pour ses problèmes est à son tour interrogé. Il raconte que le prévenu lui a été envoyé par un premier médecin, qu'il se plaignait d'être violent, que son confrère aurait souhaité une hospitalisation d'office mais que l'accusé l'a refusée. Le président demande alors si l'accusé était sincère, et le médecin confirme. Pourtant, lorsque l'avocat général pose la question de l'efficacité des médicaments prescrits autrement dit, des soins, des médicaments peuvent-ils annihiler cette dangerosité? - et de la sincérité de l'accusé, qu'il présente comme une «sincérité de façade», le médecin répond cette fois que la volonté n'était pas à l'ordre du jour car le prévenu n'est pas revenu au rendez-vous suivant.

À défaut d'une réponse basée sur une évaluation psychologique ou psychiatrique, c'est une réponse basée sur des faits (il n'est pas revenu à son troisième rendez-vous) qui est donc apportée. Le psychologue interrogé sur le même thème refusera de se prononcer sur le passé: aujourd'hui l'accusé est sincère [dans son désir de soin] mais pour avant, je ne sais pas.

Les experts, psychiatre et psychologue, ont été sollicités sur la qualité de la volonté affichée de travailler sur soi mais ont répondu de manière prudente (il faut voir les circonstances; avant on ne sait pas). C'est la dernière réponse du médecin soignant qui semble avoir clôturé le débat et particulièrement le fait que l'avocat de la défense ne le relance pas.

\section{Conclusion}

Les performances des experts «psy» au tribunal ne constituent pas des formes dégradées de l'expertise vis-à-vis d'un rapport écrit qui représenterait, lui, la forme aboutie de la pratique. La destination finale de l'expertise judiciaire au pénal est d'être entendue par le jury, de l'éclairer et de lui fournir des éléments pour juger. Lorsqu'il interroge et diag- 
nostique en garde-à-vue, dans son service de médecine médico-légale, qu'il rédige son rapport ou qu'il participe aux débats à l'audience, l'expert agit dans des contextes, des mondes d'action, comprenant des contraintes - de temps, de respect de formes... - qui sont indissociables de sa pratique. Il écrit sur commande, avec des missions, pour des lecteurs bien précis, les magistrats, et non comme il le ferait à l'adresse de collègues pour un congrès scientifique. Des magistrats qui ont des attentes, plus ou moins explicites, avec qui il entretient des relations sociales.

Le rapport n'est qu'un des éléments sur lesquels s'appuie l'expert - et d'une manière lâche, contingente - lors de sa prestation à l'audience. Il se réfère également à « ce que dit la science», «ce qu'on sait par ailleurs», son expérience; il répond aux questions qu'on lui pose, rebondit sur ce qui a été dit précédemment ou écrit la veille dans le journal. Il parle avant, après ou en l'absence d'autres experts «psy», des enquêteurs de personnalité, sous la conduite d'un président plus ou moins intéressé par l'examen de la personnalité. Des observations isolées au tribunal correctionnel suffisent à l'observateur pour percevoir que, dans un contexte d'action très différent, les expertises «psy» ne prennent pas la même part au jugement, que la performance est substantiellement différente parce que les conditions de son déroulement le sont.

Paradoxalement, l'analyse de l'expertise «psy» judiciaire s'est peu attachée à ces performances alors qu'elles constituent la raison d'être de tout le travail qui précède. Les psychiatres par exemple, dans leurs revues, proposent, à côté des analyses cliniques de cas, nombre de considérations sur les réformes législatives, l'histoire de l'expertise, ce que l'expertise ou une bonne loi devraient être, mais rien sur l'audience, lieu majeur de leur pratique. Sociologues et sociolinguistes ont pareillement ignoré l'audience ${ }^{21}$.

Cet article entendait remédier à cette lacune et présenter ce qu'il en est, dans les faits, de l'observation médico-psychologique au procès pénal en partant non seulement de l'observation des pratiques des acteurs, sous l'angle de «l'action en train de se faire», du social ou du judiciaire en acte, mais également en étant attentif aux mots pour le dire et le faire. Il ressort de ces analyses premièrement que le quotidien des experts n'est pas plus, au regard de l'histoire - contrairement à ce que les débats et les réformes mettent en exergue, d'évaluer la dangerosité ou la responsabilité au regard de son double dichotomique, l'irresponsabilité. Dans la grande majorité des procès, professionnels judiciaires et «psy» font, ensemble, autre chose. Ils débattent de lélément moral du crime, ils tentent de cerner l'engagement de la volonté, la réflexivité, le degré de conscience de l'inculpé. Ils résolvent au jour le jour la question de savoir si la consommation de drogues ou d'alcool constituent des circonstances atténuantes. Ils cherchent à savoir si, en son for intérieur, l'accusé pouvait ignorer qu'il commettait un crime ou les conséquences de ses actes. Ce faisant, ils produisent des définitions pratiques de la responsabilité.

Les experts «psy» ne sont plus majoritairement, loin s'en faut, requis pour se prononcer sur la réadaptabilité, la curabilité, la dangerosité, sur la présence ou l'absence de troubles mentaux et leur compétence n'est plus limitée par l'absence de tels troubles. Un consensus semble exister entre tous les acteurs professionnels du procès, sur l'intérêt et la pertinence d'examiner les ressorts de la psyché des accusés au regard de leur histoire œdipienne, de leur scolarité, de l'histoire de leurs relations amoureuses et ceci tout à la

21 Les grands axes des recherches sur l'expertise au tribunal ont été présentés dans Sicot (2006). 
fois pour comprendre les ressorts de l'acte, juger de la culpabilité et décider du quantum de peine.

\author{
Sébastien Saetta \\ François Sicot \\ Tristan Renard \\ LISST \\ Maison de la recherche \\ 5, allées Antonio Machado \\ 31058 Toulouse Cedex 9 \\ France \\ sicot@univ-tlse2.fr
}

\title{
Bibliographie
}

ANCEL M., 1952, Le procès pénal et l'expertise scientifique des délinquants, Melun, Imprimerie administrative. ANCEL M., 1983, La défense sociale, Paris, PUF, coll. «Que Sais-je».

BORZEIX A., FRAENKEL B. (coord.), 2001, Langage et travail. Communication, cognition, action, Paris, CNRS Éditions.

CHAUVAUD F., DUMOULIN L., 2003, Experts et expertise judiciaire. France, XIX $X^{e}$ et XX $X^{e}$ siècles, Rennes, Presses universitaires de Rennes.

DANET J., 2008, La dangerosité, une notion criminologique, séculaire et mutante, Champ pénal / Penal field, nouvelle revue internationale de criminologie [En ligne], Vol V |2008, mis en ligne le 7 octobre 2008, Consulté le 23 novembre 2009. URL: [http://champpenal.revues.org/6013].

DODIER N., 1993, L'expertise médicale, Paris, Métailié.

DINGWALL R., 2000, Language, Law, and Power: Ethnomethodology, Conversation Analysis, and the Politics of Law and Society Studies, Law \& Social Inquiry, 25, 3, 885-911.

DUMOULIN L., 2000, L'expertise judiciaire dans la construction du jugement: de la ressource à la contrainte, Droit et Société, 44-45, 199-223.

DUPRET B., 2001a, L'intention en acte. Approche pragmatique de la qualification pénale dans un contexte égyptien, Droit et Société, 48, 439-467.

DUPRET B., 2001b, Le droit en action et en contexte. Ethnométhodologie et analyse de conversation dans la recherche juridique. Présentation, Droit et Société, 48, 343-348.

GARFINKEL H., 2007, Recherches en ethnométhodologie, Paris, PUF.

GARLAND D., 2001, The culture of control, New York, Oxford University Press.

KOMTER M., 2001, La construction de la preuve dans un interrogatoire de police, Droit et Société, 48, 367-393.

MATOESIAN G., 1997, «I'm sorry we had to meet under these circumstances »: Verbal Artistry (and Wizardry) in the Kennedy Smith Rape Trial, in TRAVERS M., MANZO J.F., Law in Action. Ethnomethodological and Conversation Analytic Approaches to Law, Aldershot, Ashgate, 137-182.

MATOESIAN G., COLDREN J., 2001, Évaluer la police de proximité. Style indirect, ambiguïté et paroles rapportées dans un contexte juridique bureaucratique, Droit et Société, 48, 395-415.

ROSE N., 1989, Governing the soul. The shaping of the modern self, London, Free Association Books.

SICOT F., 2006, La maladie mentale, quel objet pour la sociologie?, Déviance et Société, 30, 2, 203-232.

TRAVERS M., MANZO J.F., 1997, Law in Action. Ethnomethodological and Conversation Analytic Approaches to Law, Aldershot, Ashgate.

UGHETTO P., WELLER J.M. (coord.), 2008, Les juges de proximité au travail. Une sociologie pragmatique et comparative, LATTS, Mission de recherche Droit \& Justice.

VRANCKEN D., MACQUET C., 2006, Le travail sur soi. Vers une psychologisation de la société?, Paris, Belin. 


\section{Summary}

The problem of evaluating the personality of suspects, which runs alongside that of individualising sentencing, is at the centre of current preoccupations known as «the new social defence' ». This article is concerned with the use of experts in a particular context, that of cases at the higher criminal courts, where the work of experts is built into a chain of different procedural steps and where the personality of the suspect is considered by several different actors. The exchanges around these questions are not so much concerned with defining dangerousness or treatability, as the moral aspect of the crime. This call for expertise shows that, in this process, those involved are constructing practical definitions of responsibility.

\section{Zusammenfassung}

Die Frage der Bewertung der Persönlichkeit von Angeklagten, die die Individualisierung von Strafen begleitet, steht im Zentrum der aktuellen Beschäftigung mit dem Thema einer neuen Sozialverteidigung. Der Beitrag beschäftigt sich mit der Leistung von Experten in einen spezifischen Kontext, den Schwurgerichten, an denen die Meinung von Experten an verschiedenen Stellen im Verlauf der Prozesse bedeutsam wird und wo die Persönlichkeit von Angeklagten von verschiedenen Akteuren thematisiert wird. Die Diskussionen, die um diese Frage kreisen, zielen aber weniger auf die Definition einer Gefährlichkeit oder Therapierbarkeit, sondern eher um die moralische Qualität des Verbrechens. Diese Perspektive auf die Praxis der Expertise zeigt, wie die Akteure im Prozessverlauf praktische Definitionen von Verantwortlichkeit konstruieren.

\section{Sumario}

La cuestión de la evaluación de la personalidad de los acusados, relacionada con la de la individualización de las penas, está en el centro de las preocupaciones de la «nueva defensa social». Este artículo analiza la competencia del experto en las audiencias de las cour d'assisses en las que la palabra del experto se integra en una cadena de secuencias y donde la personalidad del acusado es estudiada por una pluralidad de actores. Desde ese momento, el debate en torno a esa cuestión no se dirigen a definir la peligrosidad o la posibilidad de cura, sino que giran en torno al elemento moral del crimen. Esta mirada sobre el trabajo del experto muestra que, en estos procesos, los actores construyen definiciones prácticas de la responsabilidad. 